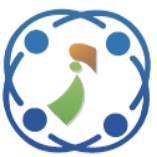

\title{
Private Browsing Forensic Analysis: A Case Study of Privacy Preservation in the Brave Browser
}

\author{
Ahmed Redha Mahlous ${ }^{1 *}$ \\ Houssam Mahlous ${ }^{2}$ \\ ${ }^{1}$ Prince Sultan University, Kingdom of Saudi Arabia \\ ${ }^{2}$ King's College London University, United Kingdom \\ * Corresponding author's Email: armahlous @psu.edu.sa
}

\begin{abstract}
The Internet and its users are in continual growth. With it grows the number of organized crimes on the Internet and the potential for individuals to carry out illegal activities. These criminals have gained more awareness of private browsing facilities, and many have found a haven in privacy designed browsers that cover up their tracks and shield their nefarious actions. The development of these privacy features has proven to be a challenge for digital forensic investigators. They strive to perform a thorough analysis of web browsers to collect artefacts relating to illegal activity to be presented as evidence to the court of law and used to convict criminals. "Brave" browser is one of the most recent and fastest-growing private browsers that, up to this point, has not been studied in-depth, and its privacy preservation functionality remains unclear. In this paper, we studied Brave's private browsing mode, examined its privacy-preserving and forensic data acquisition, and outlined the location and type of evidence available through live and post-mortem state analysis. The unique approach taken included a set of experiments that unveiled how the browser functions and showed the appropriate tools that could be utilized to extract leftover artefacts. Analysis of our results showed that despite Brave leaving no traces of browsing activity on the Hard Disk, visited URLs, images, keyword searches, and even cached videos were retrievable from the RAM, which shows that Brave is not entirely private.
\end{abstract}

Keywords: Private browsing, Web browser forensics, Forensic acquisition and analysis, Live data forensics, Postmortem forensics, Brave browser, Private browsing forensics.

\section{Introduction}

Accessing the Internet nowadays has become nearly inevitable, and web browsers remain the most popular tool to do so. The increased amount of web browser users and their aspiration to achieve paramount personal privacy has pushed developers to devise different ways to fulfil the users' need for anonymity and seclusion. One of the outcomes of this campaign was the development of private browsing modes whose main aim is to keep user's browsing sessions private from other users of the same device [1] by not retaining temporary session data. Despite this feature proving useful for people working from shared computers at work, school, and libraries, they are not the only ones enjoying the fruits of it. Cybercriminals have taken advantage of private browsing modes to clear any digital traces leftover on the machine used and leave computer forensic examiners empty-handed. The UK Office for National Statistics (ONS) estimates that around 4.5 million cybercrimes were committed in England and Wales during the year of 2018 only [2]. This further shows the vital importance of capturing and analysing digital evidence for any computer forensic investigation as it can pinpoint the source of compromise which could be the silver bullet that connects criminals and brings them to justice. We've taken it upon our shoulders to investigate Brave Browser and its private mode to try and examine the artefacts left behind, if any, from private browsing sessions and what tools can be used to extract them as well as their locations. Similar studies usually use digital forensic tools to scan the whole memory in general and search only for keywords relating to 
browsing session activity after using the browser, and this is not always accurate as sometimes browsers store session data in hex code for example rather than plain English and this will not be picked up by general keyword searches like these. For our approach, however, we decided to take it two steps further in order to search for artefacts in the right places and leave a smaller margin for error. First, we took a snapshot of the memory before installing the browser and one right after. This allowed us to pinpoint the files and folders created by Brave, which focuses our search later on as they are the most probable storage locations for Brave's browsing session data. After that, we used Brave in its normal browsing mode (not the private mode) and snapshots of the memory were taken and consequently compared with snapshots of the memory after using the browser in its private mode. This has many advantages as it allowed us to identify the behaviour of the browser in both modes and observe the differences in the types of files stored, the amount of data, the data content, and check whether Brave's private mode just simply deletes the files that would normally be left in the normal browsing mode, or whether it doesn't store them in the first place. Furthermore, we performed a live memory analysis to recover any artefacts from the RAM as well as a post-mortem analysis to retrieve them from the Hard Disk.

The remainder of the paper is organized as follows: Section 1 presents the introduction. Section 2 presents browser forensic background; then, analysis environment preparation is presented in Section 3. The forensic analysis methodology is discussed in detail in Section 4. Section 5 describes results and analysis, while section 6 presents a discussion for the results. Finally, we conclude and discuss future work in Section 7.

\section{Browser forensics background}

Web browser forensics is a branch of digital forensics that aims to identify and collect evidence and essential information related to a crime from recovered traces of browsing sessions to be used for forensic investigation purposes. Browsers store a notable proportion of user data and their browsing activities that range from cached files and visited URLs to usernames and passwords used during browsing sessions. This has led to the development of private browsing modes and consequently private browsers that claim to erase all data related to a browsing session and prevent it from persisting on the device as a way to honour the privacy of its users. Since the introduction of private modes in 2005 by
Apple Safari, many researchers went forward to test the extent of truth in these claims and whether private browsing modes actually behave as advertised and provide users with the protection they rely on and believe they have. The study [3] in 2014 defined a threat model and then conducted experiments by applying common local and remote attacks to assess the security of private browsing in the four most popular browsers: Chrome, Safari, Firefox and IE. Analysis of the results obtained brought to light a range of vulnerabilities applicable to private browsing implementations due to a couple of reasons, such as lack of control of extensions running in private mode and negligence of edge case testing. Furthermore, bookmarks and program crashes were proved to cause privacy leaks.

These four browsers were put to the test again in [4]. The experimental results made after the machine was turned off showed that private modes in Firefox, IE and Safari left traces of browsing data that are easily recoverable by using the right tools, while Chrome's Incognito did not leave any browsing artefacts behind.

In 2018, the study [5] further showed that private browsing data created by browsers such as Chrome, Firefox, IE, Safari and Opera could be retrieved from the RAM using RAM imaging or from the hard disk.

Knowing that browsers leak private browsing data is something, but the location of these artefacts is of utter importance. Researchers in [6] investigated web browser's $\log$ files which usually store cache, history and cookie files in a Windows environment. It brought to notice the limitations of methodologies in digital forensics and existing tools at that time, then proposed advanced methodology to tackle them. The study conducted also introduced a new tool, WEFA, which parses these $\log$ files and provides various functionality such as timeline analysis, user activity classification, report generation as well as recovering deleted $\log$ files. Another study [7] also observed web browser log files for Opera, Chrome, Firefox, and IE and suggested an evidence collection methodology that would help to analyse and extract information from these log files using tools like Autopsy, NetAnalysis, and Internet Evidence Finder.

So far, log files have proven to be a gold mine for private investigators, but it is not the only location where browsers leave evidence trails. Researchers in [8] examined the recoverable artefacts leftover by browsers using private browsing modes and portable browsers. The four major browsers tested were IE, Firefox, Chrome and Safari, and the results showed that most leftover artefacts were found in RAM and Orphan directories. Nihad A. Hassan in his book "Digital Forensics Basics" [9] conveys how to 
investigate web browsers and e-mail messages for forensics artefacts. It includes valuable information as to where each browser stores its cookies, history, typed URLs and cache as well as step by step analysis to extract valuable information from email headers such as the sender's geographic location.

A somewhat more general study [10] in 2019 examined the "privateness" of 30 web browsers on a Windows 10 OS. The experimental results showed that some browsers leaked browsing session data and almost all of them had keyword hits using a triagestyle keyword search. The keyword hits were mainly found in $\log$ files, free space, \$MFT and dat files.

There are various tools that could be used to extract the information left behind by browsing sessions. Researchers in [11] observe major web browser analysis tools in a Windows environment and highlights the advantages and limitations of some over the others. The study also shows that using a carving tool such as ESECarve, Internet explorer's InPrivate browsing records can be retrieved from various areas on the disk such as the database file, WebCacheV01.dat and log files. Work by Chivers [12] on InPrivate browsing mode, which claims that it prevents local storage on a computer, also revealed that recovery of browsing records is possible either from database log files or by carving records of the disk even after a machine is powered down.

Similar investigations have been made on other browsers. The research paper [13] studied three privacy-enhanced web browsers, Epic, Commodo and Dooble, and compared their private browsing modes with those of three commonly used browsers, Edge, Firefox, and Chrome, based on the number of recoverable artefacts produced and their contents. The study used FTK and Autopsy as tools to find the number of residual artefacts on Windows operated machines, and only used ten websites to generate web traffic. However, the results were inconclusive as to whether any of the two groups provided better privacy than the other.
Similarly, [14] examined Browzar, a privacypreserving Internet browser, and compared its results with Chrome and Firefox. The study was based on change monitoring, live data forensics and postmortem analysis, carried out using a set of tools primarily composed of Procmon, IEF, FTK and XWays. Based on the evidence found and analysis conducted, it was shown that out of the three browsers, Browzar left the most information behind, including files, folders, keyword searches, URLs, and pictures.

Another study [15] conducted live and postmortem analysis of the Epic Privacy Browser on Windows $7 \&$ and Windows 10 machines; the study found out that despite temporary files and folders being cleared at the end of a browsing session, there were still remnant traces that could be recovered using standard tools such as IEF and Regshot.

Despite all the research conducted on different web browsers and their private modes, we noticed a lack of attention given to Brave, a browser which prides itself in the security and privacy it provides and has over 13.5 million active users per month [16] who rely on it for their personal use. This paper aims to bridge this gap and present details that might be of use to both users and private investigators alike.

\section{Analysis environment preparations}

In this paper we studied the behaviour of Brave browser in private mode on a Windows 10 machine. The choice of Windows 10 is justified by the fact that it holds $87.82 \%$ share of the market, as shown in Fig. 1.

To do our forensic analysis, a clean environment that avoids mixing browsing artefacts was mandatory. With many options at hand to achieve this, we settled on using a virtualized environment using VMWare Fusion. Other than providing a clean environment out of the box, this choice is further justified for the following reasons:

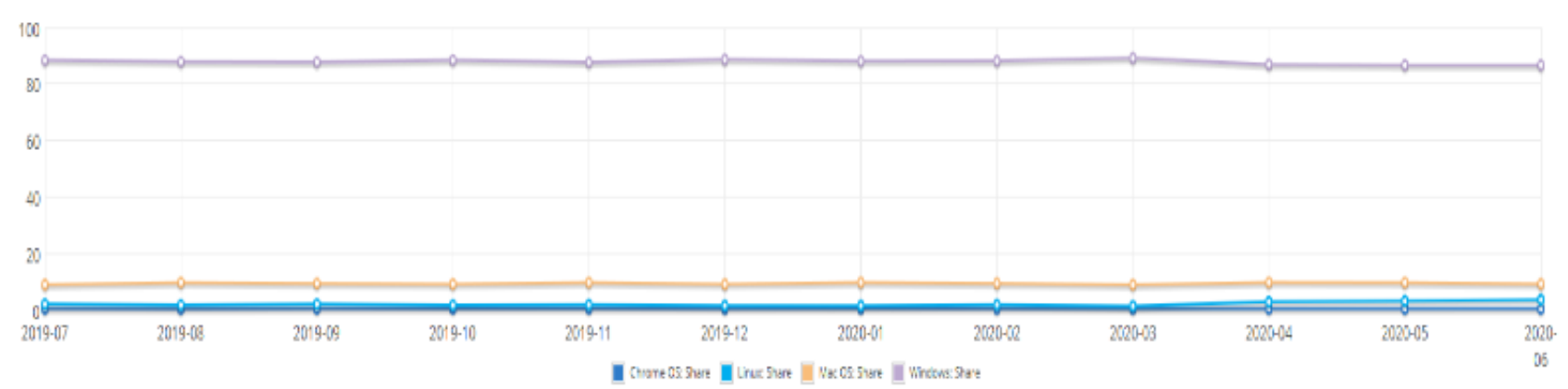

Figure. 1 Operating system share by version [17] 
1. Using virtualization will allow us to set only one base virtual machine with necessary configuration, then multiple snapshots could be taken and used later

2. Saving Experiment time, knowing that taking a snapshot would require only a few seconds.

3. Possibility to revert the machine to its initial state easily and quickly.

A Windows 10 virtual machine based on a iso image acquired from our academic software license portal [18] was used. A pre-configured 1 TB hard disk drive that was wiped according to the NIST 80088 Standard for Media Sanitization [19] and contained separate tools and evidence part was connected to a MacBook Pro computer. Its primary purpose was to run the tools and store the vmdk image and RAM (dump) for post-mortem forensic examination.

A fresh Windows 10 operating system was installed on the virtual machine. The Brave browser was then installed on the system using an installer that was transferred via a USB. Doing so ensured that the environment was kept as clean as possible, eliminating the chance of mixing the artefacts left by the default browser with the ones left by the Brave browser later on.

The following tools were installed for conducting the analysis:

- $\quad$ FTK Imager [20]

- Autopsy 4.15.0 [21].

- Regshot [22]

- Internet Evidence Finder V6.4 [23]

- WinHex [24].

To make the experiment as realistic as possible, the tasks listed below (based on the most visited websites in the UK as of July 2020 [25] were performed with the Brave browser. A keyword search (using Autopsy) was conducted before performing the tasks to rule out any possible cause of contamination. No results were found for any of the keywords from the tasks (e.g. "basic rat python", or the words from Table 1), ensuring that any results found later are not false positives.

Tasks:

1. Visit www.youtube.com, search for "basic rat python" and watch the first video.

2. Visit www.google.com, search for the keywords in Table 1 and click on one of the search results.

3. Visit www.gmail.com and sign in with an account.

4. Visit www.skysports co.uk.
Table 1. Keywords searched and visited URLs

\begin{tabular}{|l|l|}
\hline Keyword & \multicolumn{1}{|c|}{ Visited URL } \\
\hline $\begin{array}{l}\text { Basic rat } \\
\text { python }\end{array}$ & $\begin{array}{l}\text { https://www.youtube.com/watch?v= } \\
\text { tczUv_RK-fk }\end{array}$ \\
\hline Cars & $\begin{array}{l}\text { https://www.daimler.com/products/ } \\
\text { passenger-cars/ }\end{array}$ \\
\hline Malware & $\begin{array}{l}\text { https://searchsecurity.techtarget.co } \\
\text { m/definition/malware }\end{array}$ \\
\hline $\begin{array}{l}\text { Forensic } \\
\text { tools }\end{array}$ & $\begin{array}{l}\text { https://techtalk.gfi.com/top-20-free- } \\
\text { digital-forensic-investigation-tools- } \\
\text { for-sysadmins/ }\end{array}$ \\
\hline beach & $\begin{array}{l}\text { https://www.agoda.com/coco-palm- } \\
\text { beach-resort-spa/hotel/phu-quoc- } \\
\text { island-vn.html?cid=1844104 }\end{array}$ \\
\hline
\end{tabular}

5. Visit www.amazon.co.uk, search for "MacBook" and view the results.

6. Visit www.bbc.co.uk.

After running these tasks for 48 hours on Brave browser's private mode, a copy of the RandomAccess Memory (RAM) was captured using FTK Imager (version 3.1.1.8), prior to shutting down the machine. The VMWare machine was then shut down, and an image of it was acquired using Autopsy [for the post-mortem analysis].

\section{Forensic analysis methodology}

\subsection{System changes after installing brave browser}

To track changes to the system registry as a result of installing the browser, Regshot was used to take a snapshot of the registry before installation (Fig. 2.).

A second snapshot was taken after installing the browser and compared with the first one. Regshot generates a report of the results, showing the new files and folders that were added to the registry key. Searching for "brave" in the report reveals some of the changes that are definitely related to the

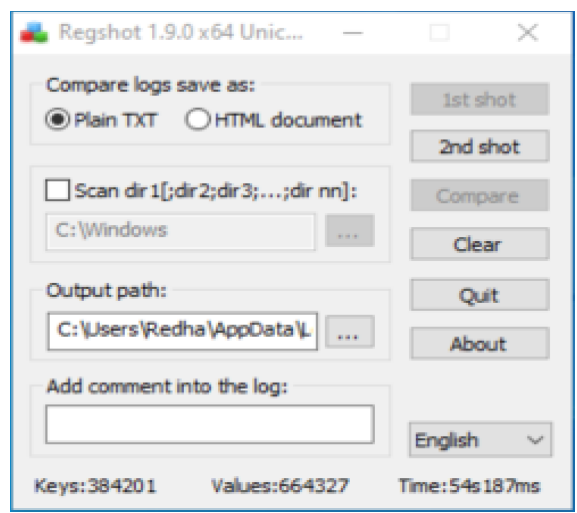

Figure. 2 Regshot $1^{\text {st }}$ shot registry capture (before brave installation) 


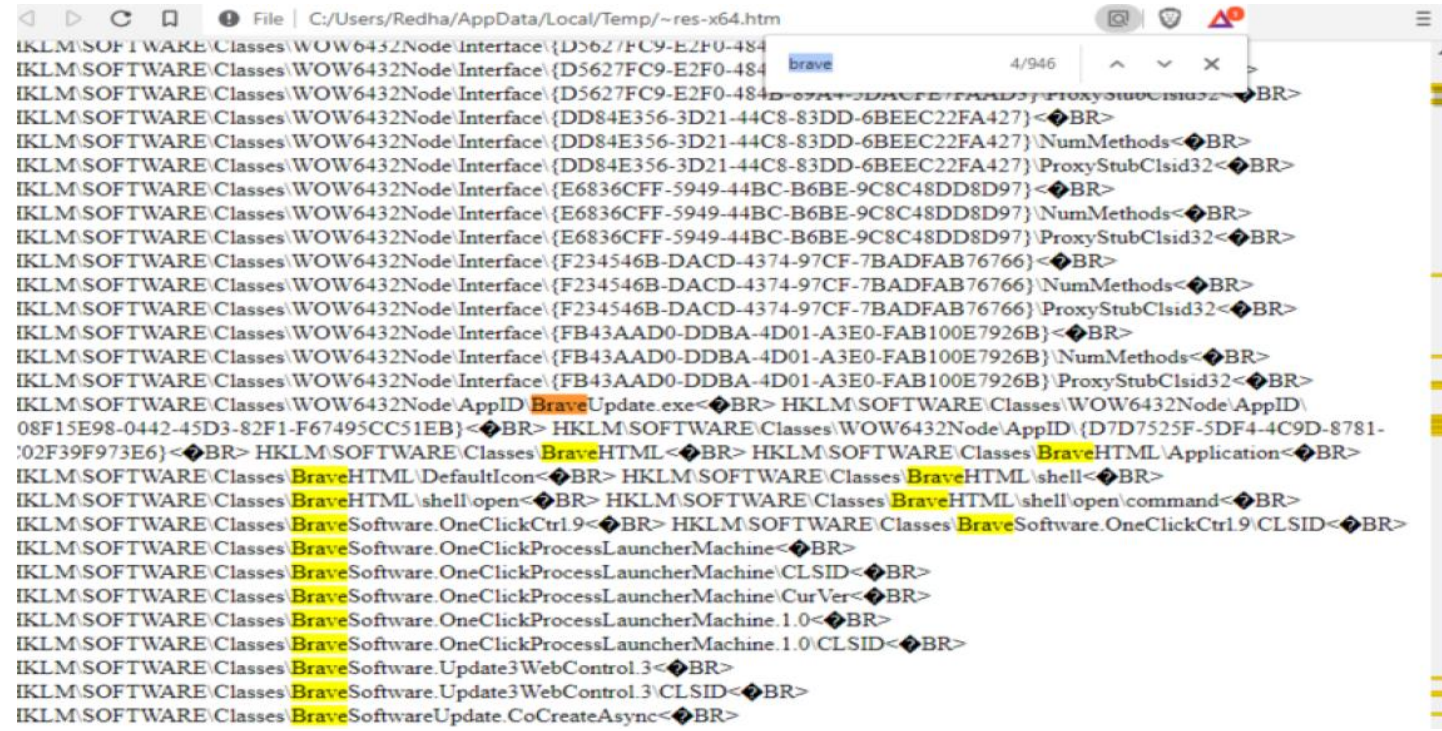

Figure. 3 Windows registry comparison results for brave browser

\begin{tabular}{|c|c|c|c|c|c|c|c|c|c|c|c|c|c|c|c|c|c|c|}
\hline fiew Tools S & Specialist & \multicolumn{3}{|c|}{ Options } & \multicolumn{6}{|c|}{ Window Help } & & \multirow{2}{*}{\multicolumn{4}{|c|}{ A $A$ A $B$}} & \multirow{2}{*}{\multicolumn{2}{|c|}{$\rightarrow \rightarrow+\Rightarrow \mid 23 \geqslant$}} \\
\hline 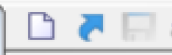 & 0 & - 国 & + & & & 国实 & 龱 & 四 & $3 \begin{array}{l}101 \\
01\end{array}$ & & & की & & & & & & \\
\hline \multicolumn{19}{|c|}{ memdump.mem } \\
\hline Offset & 0 & 1 & 2 & 3 & 4 & 5 & 6 & 7 & 8 & 9 & A & B & c & D & $\mathbf{E}$ & $\mathbf{F}$ & ANSI ASCII & A \\
\hline $00583 \mathrm{E} 60$ & 00 & 61 & 00 & 76 & 00 & 65 & $4 \mathrm{E}$ & 02 & 10 & A9 9 & 48 & 88 & 39 & 04 & 65 & 00 & a $v$ eN $e H^{\circ} 9$ e & \\
\hline $00583 E 70$ & 72 & $7 \mathrm{E}$ & 00 & 00 & ED & FF & 27 & 79 & 11 & EO & $\mathrm{EE}$ & 3B & 00 & 00 & 00 & 66 & I fy"y ai; & \\
\hline 00583E80 & 88 & 01 & 75 & $\mathrm{CB}$ & 02 & 74 & 18 & 01 & FE & $\mathrm{C} 3$ & 47 & 01 & 80 & 3F & 80 & 60 & $\because$ uE $t$ pÁG $\in$ ? & \\
\hline $00583 E 90$ & 00 & 44 & D4 & $\mathrm{AB}$ & $\mathrm{Cg}$ & 00 & 00 & 00 & 06 & 74 & 02 & 00 & 90 & 03 & 58 & 00 & DŌ $\approx \hat{E}$ & \\
\hline O05B3EAO & 59 & 02 & 9A & 72 & 46 & F7 & 52 & 96 & E7 & ED & 74 & 00 & FF & $6 \mathrm{C}$ & $\mathrm{Cl}_{1}$ & AF & 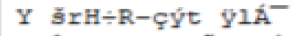 & \\
\hline O05B3EBO & $6 \mathrm{~F}$ & $9 \mathrm{c}$ & $\mathrm{CA}$ & Fo & B4 & 88 & 96 & 17 & 72 & $1 \mathrm{C}$ & FA. & $\mathrm{C}_{3}$ & A.O & C6 & 01 & $\mathrm{Ce}$ & 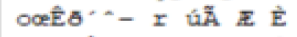 & \\
\hline 005B3ECO & 46 & 73 & $\mathrm{DF}$ & BD & $\mathrm{C} 2_{2}$ & B4 & BF & 07 & 07 & 87 & 02 & $4 \mathrm{~F}$ & 00 & 13 & 40 & E5 & FsB Ã $\bar{A} \neq 0$ Qá & \\
\hline OO5B3EDO & Bo & $3 \mathrm{~A}$ & OB & 3D & 00 & 48 & $7 \mathrm{C}$ & 00 & oc & $2 \mathrm{C}$ & 01 & 01 & 00 & 55 & 00 & 73 & $\therefore=$ HI & \\
\hline O0583EEO & $3 F$ & 06 & B.4 & 00 & 00 & 70 & 84 & 07 & $3 F$ & 14 & F3 & $\mathrm{BD}$ & 00 & 00 & 00 & 04 & $?=\mathbf{P}_{\mu} ?$ of & \\
\hline O0583EFO & 20 & 00 & 00 & $7 \mathrm{~B}$ & 05 & B9 & D4 & 96 & $\mathrm{cg}$ & 32 & 03 & 00 & 90 & 68 & 74 & $\mathrm{BB}$ & $t^{-}{ }^{\circ} \mathrm{O}-\mathrm{E} 2$ & \\
\hline $00583 F 00$ & $3 F$ & 77 & 77 & 77 & $2 \mathrm{E}$ & 67 & $6 \mathrm{~F}$ & $6 \mathrm{~F}$ & 67 & $6 \mathrm{C}$ & 65 & $2 \mathrm{E}$ & 63 & $6 \mathrm{~F}$ & $6 \mathrm{D}$ & $2 \mathrm{~F}$ & PWWw - google.com/ & \\
\hline $00583 F 10$ & 73 & 65 & 61 & 40 & 00 & 00 & 00 & 72 & 63 & 68 & $3 F$ & 71 & 3D & 62 & 65 & 61 & rch?q=bea & \\
\hline $00583 F 20$ & 63 & 68 & 26 & 73 & 61 & 66 & 65 & 3D & 73 & 74 & 72 & 69 & 63 & 74 & 26 & 73 & ch [safe=strictss & \\
\hline $00583 F 30$ & $4 \mathrm{~A}$ & $3 F$ & 3D & $6 \mathrm{C}$ & $6 \mathrm{E}$ & $6 \mathrm{D}$ & 73 & 26 & 00 & 00 & 00 & 02 & 74 & 62 & $6 \mathrm{D}$ & 3D & $J_{\mathrm{P}}=1 \mathrm{nms} \mathrm{s} \quad \mathrm{tbm}=$ & \\
\hline $00583 F 40$ & 69 & 73 & 02 & 01 & 3D & 58 & 26 & 76 & 65 & 64 & 3D & 32 & 61 & 68 & 55 & $4 \mathrm{~B}$ & is $=\mathrm{X} s$ ved $=2$ ahuK & \\
\hline $00583 F 50$ & 45 & 77 & 69 & $5 e$ & $2 \mathrm{D}$ & 36 & 36 & 53 & 30 & 35 & 72 & 71 & 41 & 00 & 00 & 00 & Ew1X-66505xqA & \\
\hline $00583 \mathrm{~F} 60$ & 00 & 68 & 58 & 46 & 78 & $6 \mathrm{~F}$ & 55 & $4 \mathrm{~B}$ & 48 & 57 & 74 & 78 & 43 & 51 & 34 & 51 & hXFXOUKHW $t \times C O 40$ & \\
\hline
\end{tabular}

Figure. 4 WinHex memory analysis results (keyword "beach" revealed)

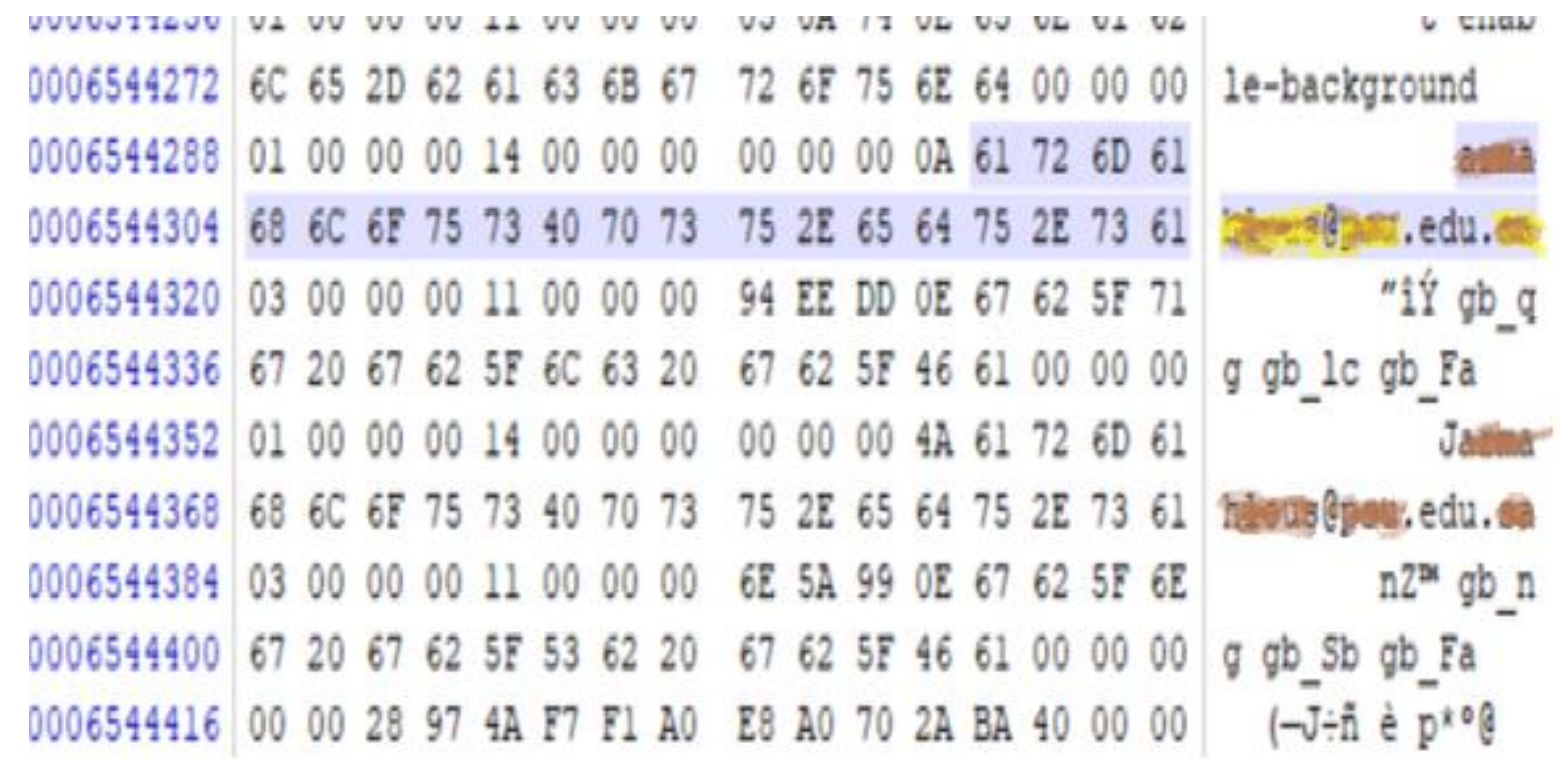

Figure. 5 WinHex memory analysis results (email revealed) 
installation, as shown in Fig. 3.

\subsection{Live acquisition and analysis}

\subsubsection{Memory acquisition}

Where live acquisition is possible, memory analysis can reveal valuable information such as decrypted programs, usernames and passwords, chat window contents, and form field entries. For this experiment, FTK Imager was used to obtain a dump of the memory contents after completing all the Internet activities and before closing the browser. The dump files were stored in an external 1TB hard disk drive for analysis.

\subsubsection{Memory analysis}

The following forensic tools were used to search for artefacts within the memory dump:

\section{a) WinHex:}

RAM analysis with WinHex revealed that some residual traces remain of email addresses, keyword searches, and many more, as shown in Fig. 4 and Fig. 5

\section{b) Internet evidence finder:}

Internet Evidence Finder (IEF) was used to search for evidence relating to the browser-specific search keywords from Table 1. IEF allows us to refine the search by choosing the type of artefacts under investigation as shown in Fig. 6 (a). Fig. 6 (b). shows IEF running the search process.

IEF's RAM analysis revealed further evidence, as shown in Fig. 7 and Fig. 8.

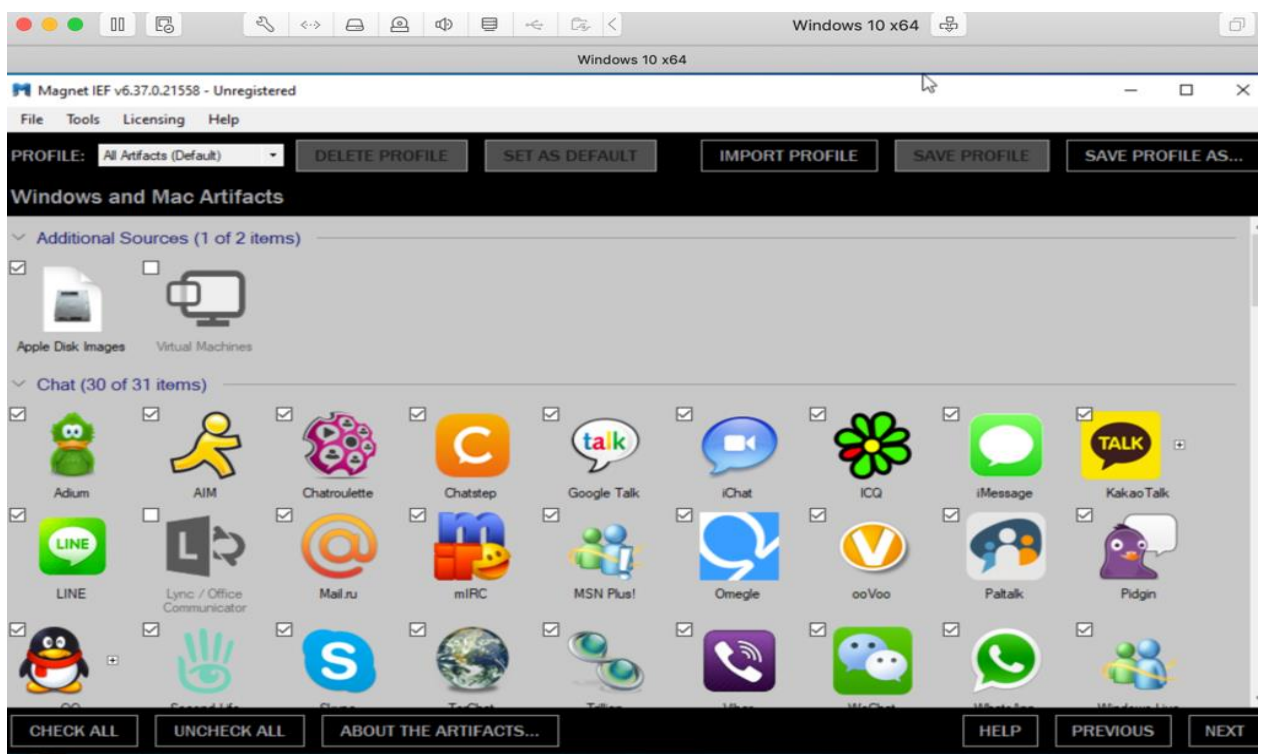

(a)

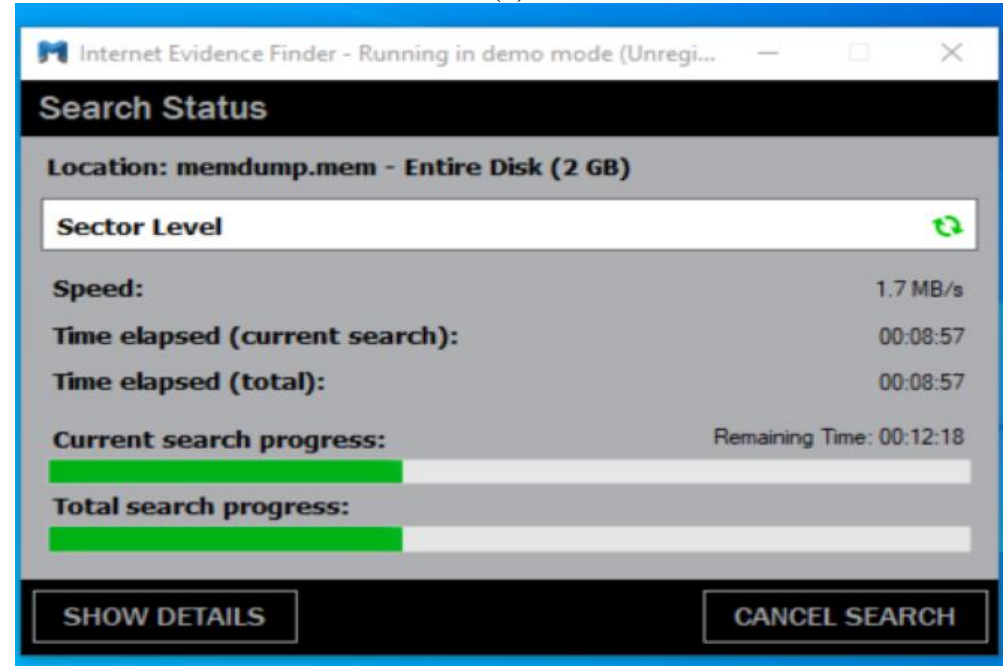

(b)

Figure. 6: (a) The search category artifacts selection in IEF and (b) RAM search process in IEF 


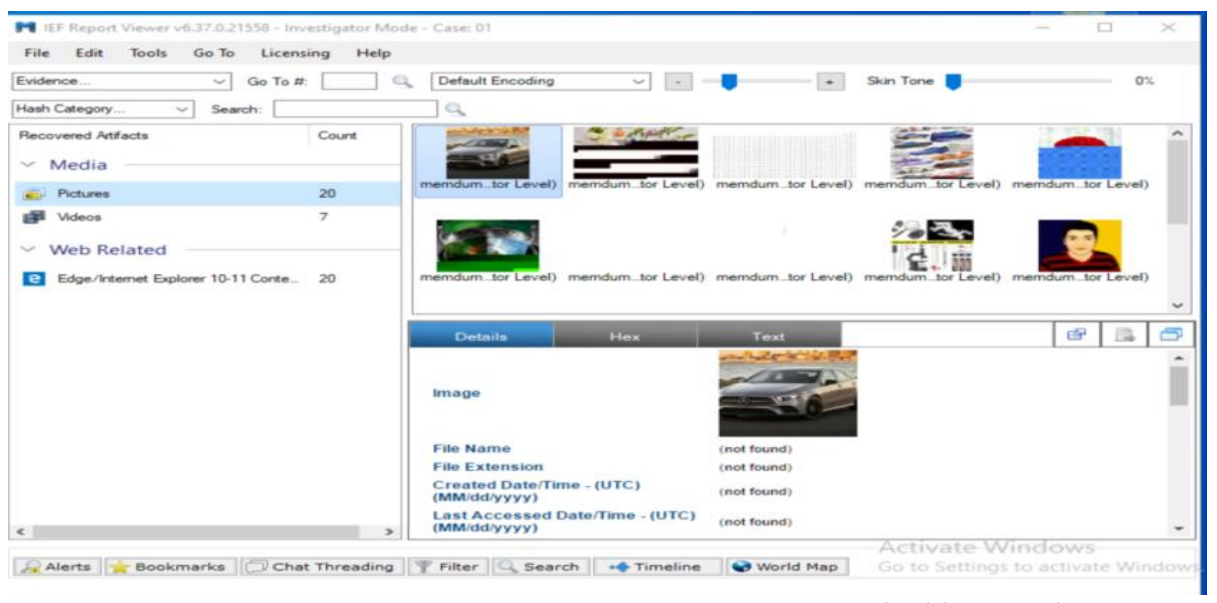

Figure.7 IEF discovery of picture of car related to the searched keyword "cars"

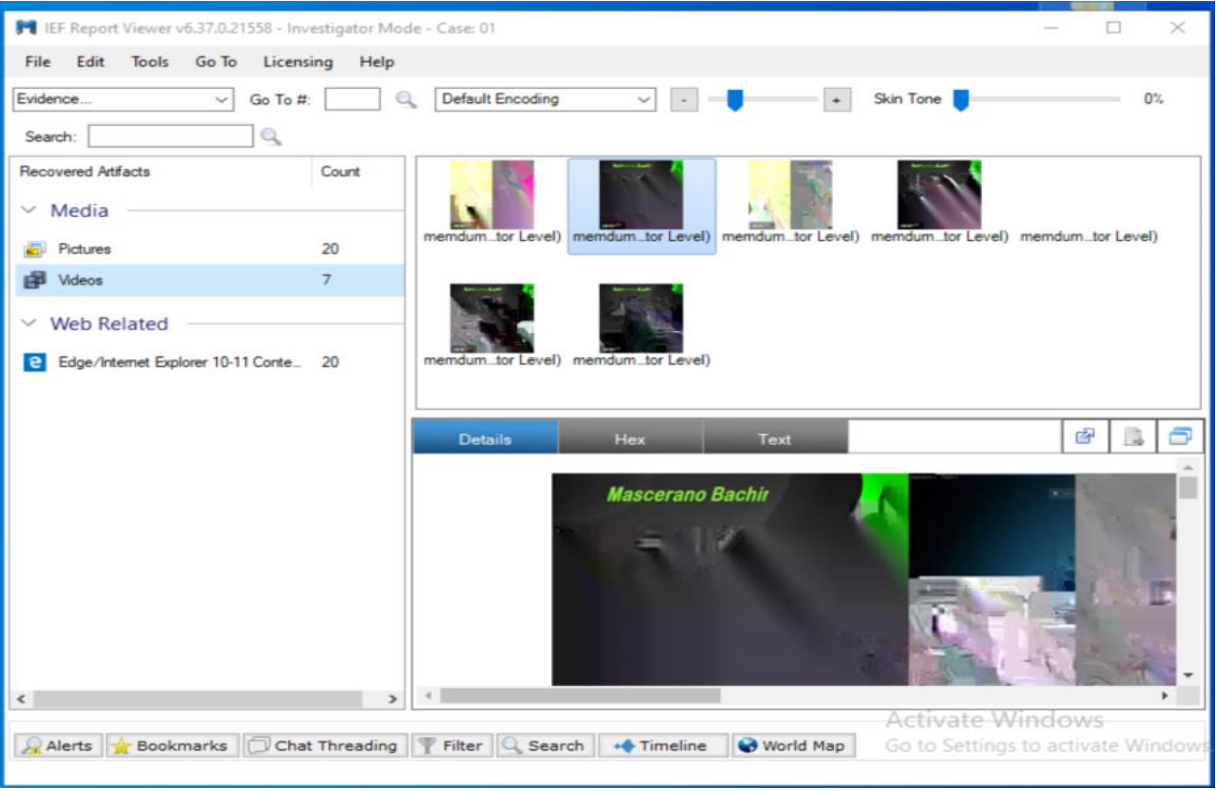

Figure. 8 IEF discovery of traces of the watched video related to the searched keyword "basic rat python"

\section{c) Autopsy software:}

Similar results were retrieved with Autopsy. Searching for websites and some of the keywords mentioned in section 3 (www.bbc.com, www.skysports.com, "basic rat python") allowed us to extract residual artefacts left by our browser in a hidden file called "pagefile.sys", which is used by the operating system to reduce the workload on the physical memory (RAM) and allow it to perform smoothly [26]; the results are shown in Figs. 9 (a), 9 (b), and 9 (c). This highlights the importance of using multiple forensic tools, since one tool may reveal more information than another. Using multiple tools also allows for the cross-validation of detected artefacts.

\subsubsection{Post-mortem data acquisition and analysis}

Forensic investigators frequently conduct postmortem analyses on disk images of devices that have been powered off. In many cases, this is the only option, since it is not always possible to have a forensic examiner at hand to perform a live acquisition. Moreover, a seized device may not be immediately examined due to delays in processing, or because of a shortage of forensic examiners compared to the number of devices waiting to be examined. It is thus unrealistic and impractical to keep seized devices powered on. Powering off a device also reduces the risk of the data being modified (either accidentally or deliberately) and isolates it from the network to prevent any attempts to wipe it remotely, among other benefits.

Two post-mortem experiments were conducted, one on a disk image obtained after a normal browsing session and the other after a private browsing session. 


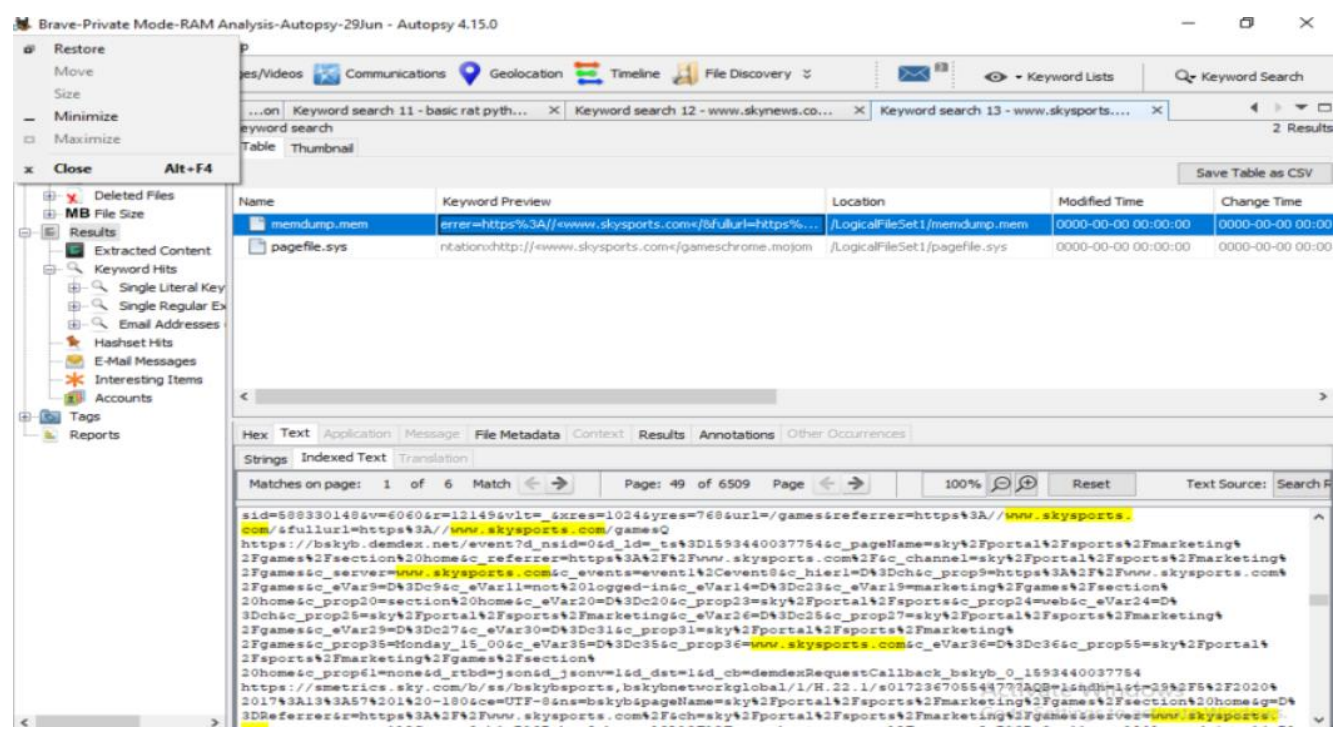

(a)

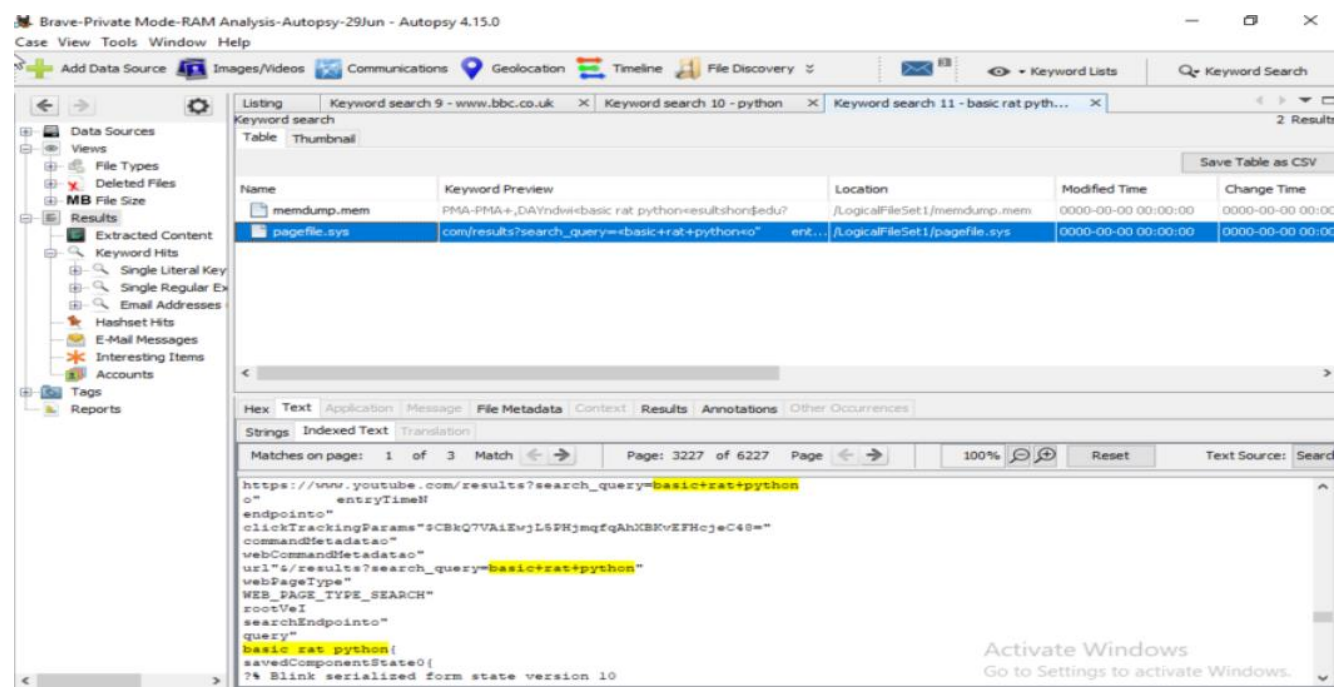

(b)

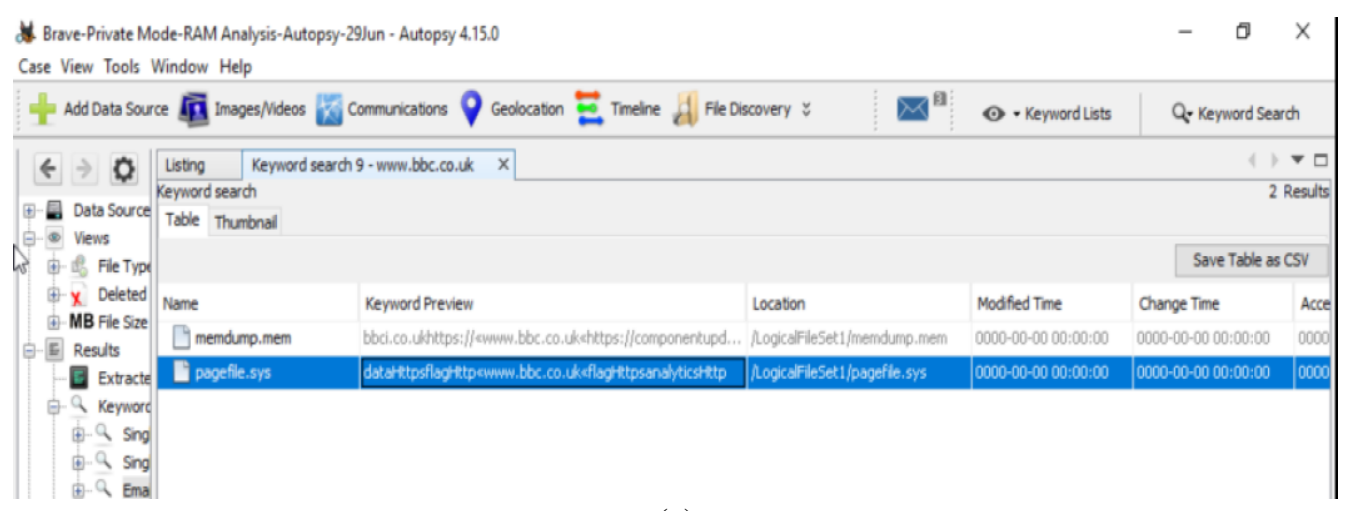

(c)

Figure. 9: (a) Autopsy keyword search result for "www.skysports.com", (b) autopsy keyword search result for "basic rat python", and (c) autopsy keyword search result for "www.bbc.co.uk"

The virtual machine was shut down after each session following the standard method, mimicking normal user behaviour. Two disk images of the virtual machine (for normal browsing mode and private browsing mode) with Expert Witness Format extension (E01) were acquired using FTK imager. The aim of the first experiment (normal browsing mode) was to identify where the browser normally 


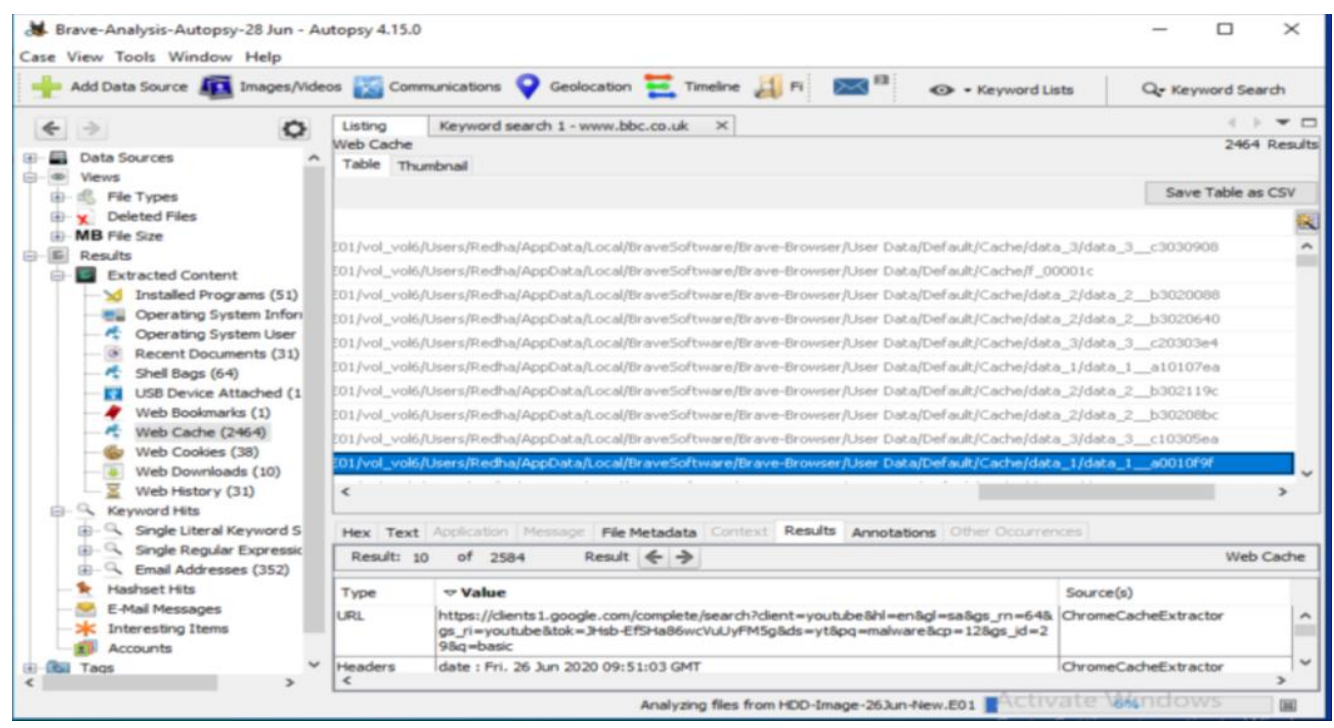

Figure. 10 Keyword search results for "basic rat python"

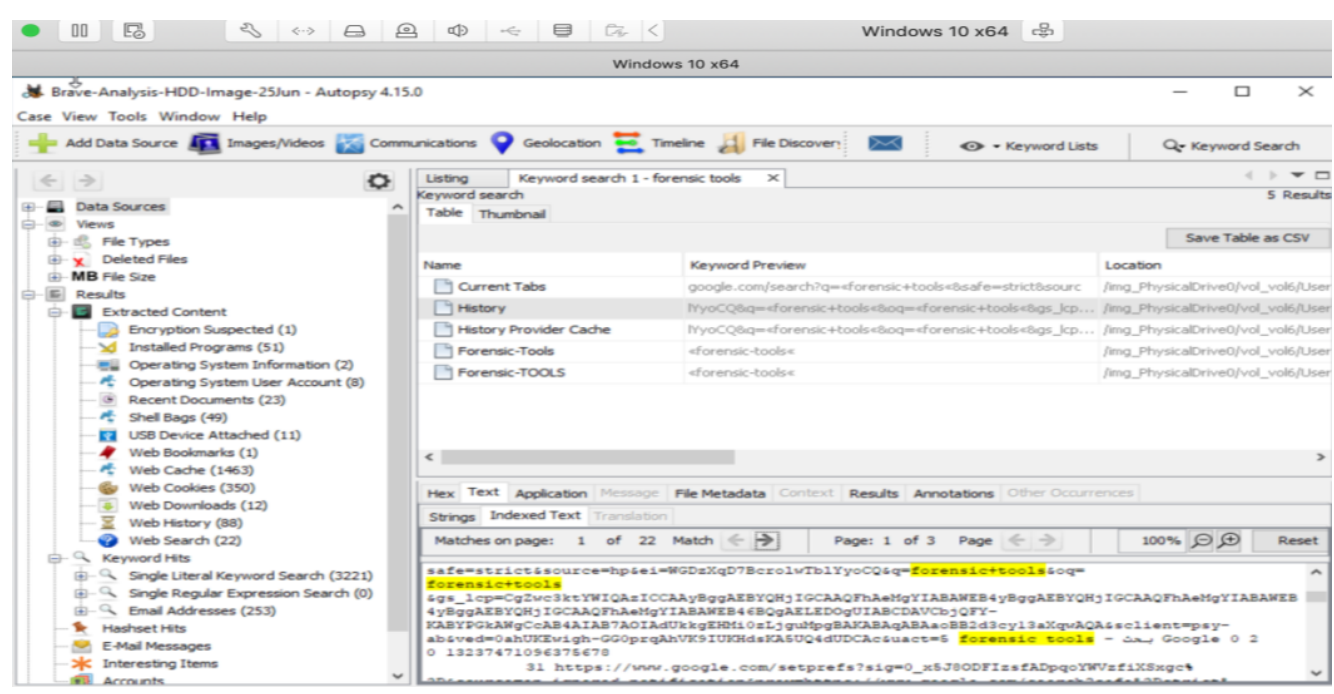

Figure. 11 Keyword search results for "forensic tools"

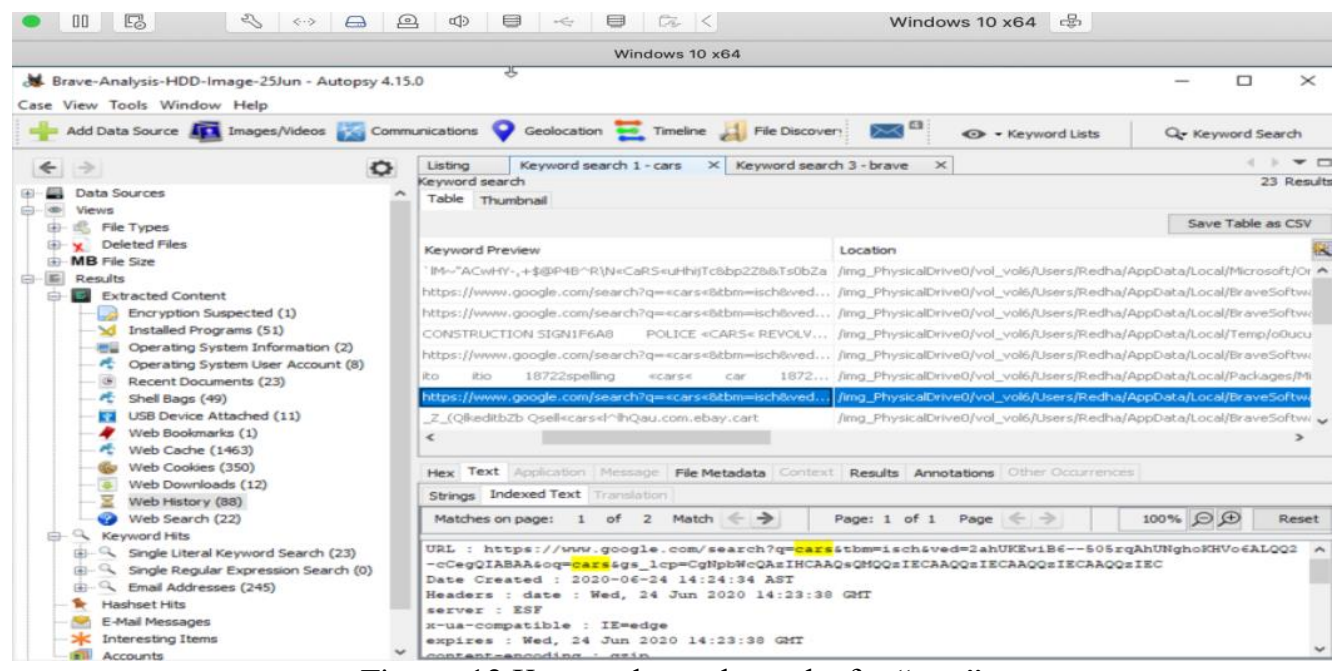

Figure. 12 Keyword search results for "cars"

stores its files, and the aim of the second experiment (private browsing mode) was to see what files were left behind if any.
In the first experiment, Autopsy was used to conduct a keyword search for the URLs visited. The result contained many hits, and it was evident that the 


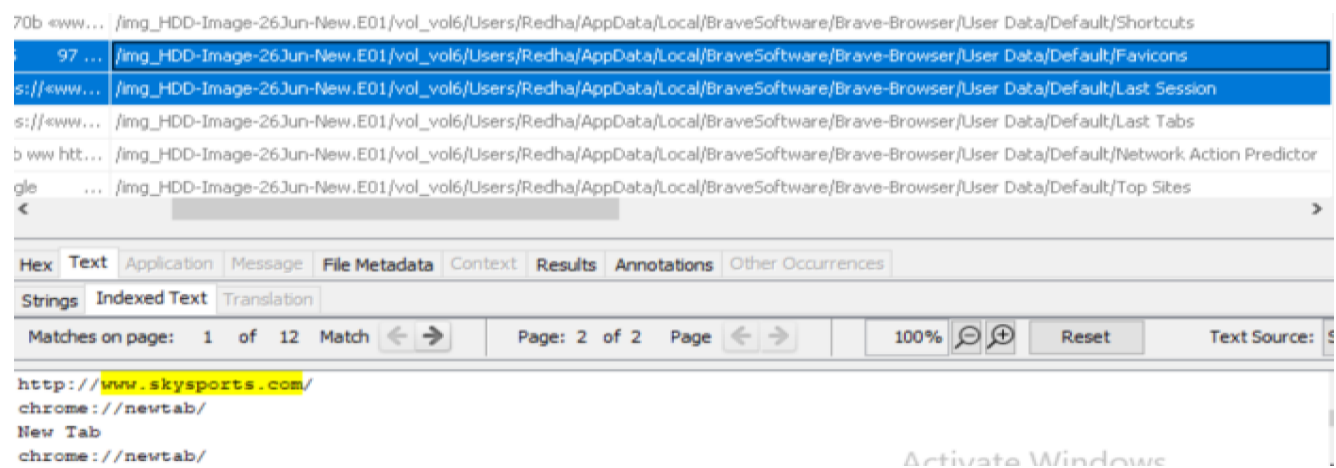

Figure. 13 Keyword search results for "www.skysports.com"

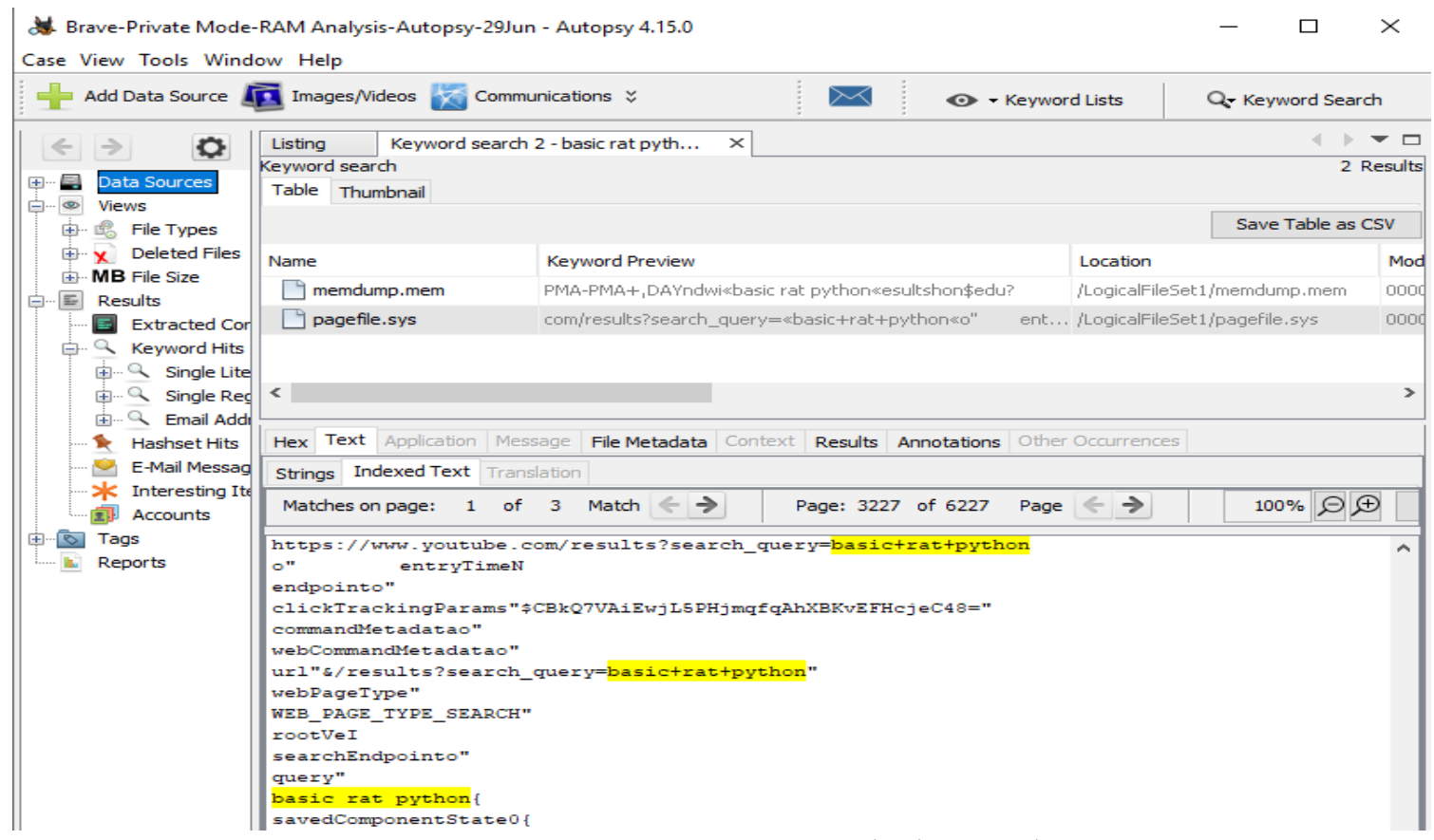

Figure. 14 Keyword search results for "basic rat python"

browser stores browsing history in the "/users/username/AppData/BraveSoftware/BraveBrowser/User Data/Default/Cache" folder as shown in Figs. 10-14.
In the second experiment the same keyword search was conducted on the image obtained after the private browsing session. No results were found, as shown in Figs. 15-17.

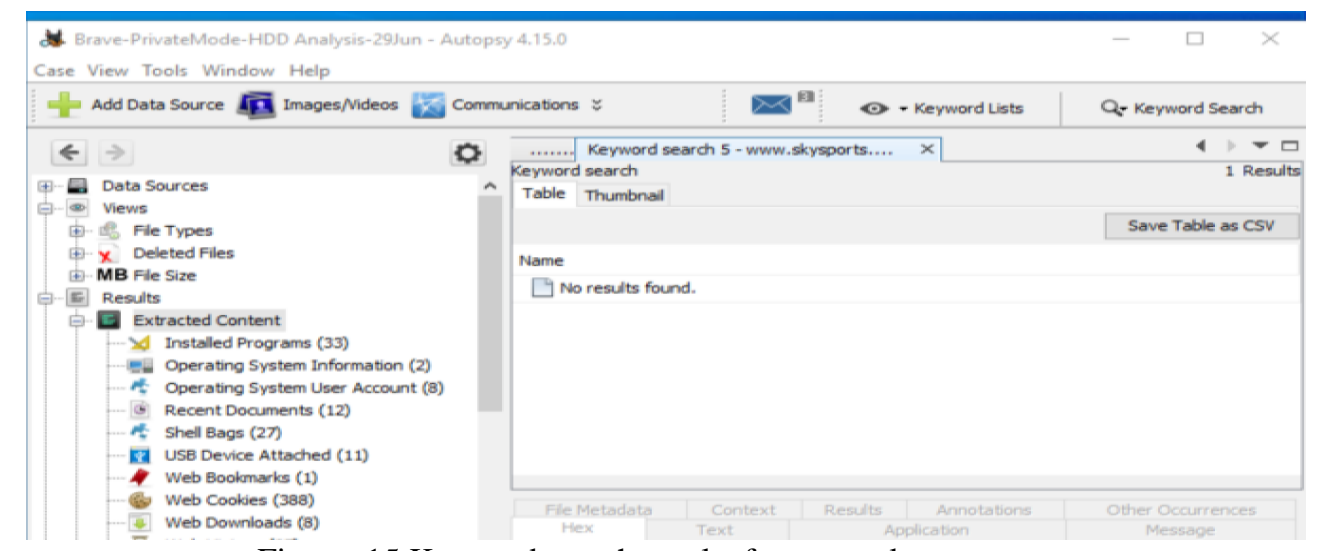

Figure. 15 Keyword search results for www.skysports.com 
Table 2. Task related artefacts found on RAM and hard disk

\begin{tabular}{|c|c|c|c|c|}
\hline \multirow[t]{2}{*}{ Task } & \multicolumn{2}{|c|}{ Hits } & \multirow{2}{*}{$\begin{array}{l}\text { Artefacts } \\
\text { found }\end{array}$} & \multirow{2}{*}{$\begin{array}{l}\text { Tools } \\
\text { used }\end{array}$} \\
\hline & RAM & $\begin{array}{l}\text { Hard } \\
\text { Disk }\end{array}$ & & \\
\hline $\begin{array}{l}\text { Watch a } \\
\text { YouTube } \\
\text { video }\end{array}$ & $\checkmark$ & $x$ & $\begin{array}{l}\text { video, } \\
\text { URLs }\end{array}$ & IEF \\
\hline $\begin{array}{l}\text { Google } \\
\text { search: } \\
\text { "beach", } \\
\text { "cars", } \\
\text { "malware" } \\
\text { "forensic } \\
\text { tools" }\end{array}$ & $\checkmark$ & $x$ & $\begin{array}{l}\text { images, } \\
\text { keywords, } \\
\text { URLs, }\end{array}$ & $\begin{array}{l}\text { WinHex, } \\
\text { Autopsy }\end{array}$ \\
\hline $\begin{array}{l}\text { Visit } \\
\text { www.skys } \\
\text { ports.co.uk }\end{array}$ & $\checkmark$ & $x$ & URLs & Autopsy \\
\hline $\begin{array}{l}\text { Visit } \\
\text { www.gmai } \\
\text { l.com and } \\
\text { sign in }\end{array}$ & $\checkmark$ & $x$ & emails & WinHex \\
\hline $\begin{array}{l}\text { Search for } \\
\text { "macbook" } \\
\text { in } \\
\text { www.amaz } \\
\text { on.co.uk }\end{array}$ & $\checkmark$ & $x$ & $\begin{array}{l}\text { URLs, } \\
\text { images }\end{array}$ & Autopsy \\
\hline $\begin{array}{l}\text { Visit } \\
\text { www.bbc.c } \\
\text { o.uk }\end{array}$ & $\checkmark$ & $x$ & $\begin{array}{l}\text { keywords, } \\
\text { URLs }\end{array}$ & Autopsy \\
\hline
\end{tabular}

\section{Results and analysis}

Table 2 summarizes the results of our experiments obtained through a set of tasks done using Brave's private mode. As it can be seen, a live memory analysis of the RAM can be really rewarding as different types of artefacts, including URLs, emails, images, and even videos, could be recovered. On the contrary, a post-mortem analysis would lead to a dead end for forensic investigators as Brave manages to clear all data and information related to its private browsing sessions from the hard disk.

\section{Discussion}

In this paper we found that after installing Brave browser on Windows 10, a number of files are created in "/users/username/AppData/BraveSoftware/BraveBrowser/User Data/Default/ Privacy Browser directory. Brave browser also creates a default folder which contains temporary files and folders used when the browser is launched and is deleted on closure. However, this is not enough to hide all traces of browser activities. Some information related to the browser's activities is left behind on the RAM, and they can be retrieved when doing memory acquisition and analysis using some standard tools. Those artefacts are left in both the memory dump and "pagefile.sys" file, which are both great places for forensic investigators to search for evidence. Many artefacts have been recovered in our experiments such as typed URLs, pictures and keyword searches. These artefacts are similar to the artefacts left using Browzar [12] and Epic [13], which are both privacyenhanced browsers. The files and folders created temporarily by these browsers got deleted at the end of each browsing session, but the data was still readily available and retrievable using digital forensic tools. Incognito, Chrome's private mode, as well the Comodo Dragon browser also produced similar results in [11], and their artefacts were found in locations nearly identical to the location of Brave's artefacts. The most probable reason for the similarity of results between the five browsers, Epic, Browzar, Commodo Dragon, Chrome, and Brave, is that they are all built on the open-source Chromium browser platform, and this explains why they all store data in similar places and in a similar way even though their functionality differs. This might show that there is a weakness in the structure of Chromium-based browsers and there is a need for improvement in the amount and content of data related to browsing sessions stored in the RAM by these browsers in their normal and private modes. Despite the similarities, Brave is the only browser whose private mode managed to leave no traces on the Hard Disk. The post-mortem analysis we conducted proved this and could be deemed accurate as two experiments were carried out; the first was using the normal browsing mode to locate the files and folders Brave normally stores its browsing session data in, and the second was using Brave's private mode and then searching the previously identified files and folders as well as scanning the whole memory using the most commonly used and standard digital forensic tools in a search for any leftover browsing session data. The acquisition and analysis of the drive image have shown no traces of the user's browsing activities, and no hits were made in the second experiment.

Indeed, seizing a suspect's running computer, with Brave browser open or minimized will be a great source of artefacts if a live memory dump is done before shutting down the system or closing the browsing session as demonstrated in our experiments, but unfortunately, that is not always the case.

\section{Conclusion and future work}

Brave browser claims to provide protection to user's privacy when online and guarantees to clear all traces of browsing history on closure. In this paper, 
we presented the forensic acquisition and analysis of Brave browser and looked into how its private mode preserves and protects the user's privacy to test the extent of truth for these claims. The series of experiments we performed have shown that some artefacts related to browser activities are still available on the RAM even after ending a browsing session and can be retrieved by forensic investigators using the right tools, but none remain on the hard disk. The approach taken in identifying the files and folders before performing the search has left a minimal margin for error in our results. The artefacts retrieved from the RAM were more than what would have been found if a standard plain English keyword search approach was used, and despite the focused search, there were still no hits on the Hard Disk. This new method would be recommended in future researches in this area as it is more accurate and would also help locate the storage locations of other poorly documented or newly created browsers.

Our study concluded that Brave browser does deliver on local privacy with turning off the machine after a private browsing session being the only caveat. The storage locations for browsing session data in both normal and private browsing modes were identified, and the type and content of leftover artefacts were outlined. These results are useful to forensic investigators seeking to recover web browser's activities of suspected users and could act as a basis for future research done on Brave and other browsers alike. The observations and the proposed approach could also be instrumental for future computer forensic investigations and to developers seeking improvement to the degree of privacy offered by their browsers.

Despite the thorough examination conducted, some research windows relating to the privacy provided by Brave Browser remain open for further investigation. For instance, running these experiments using physical devices rather than virtual machines would give more insight into the behaviour of Brave browser on a real testbed. Another area of interest would be studying and comparing Brave Browser with TOR browser, the number one Dark web browser [27], in an attempt to ascertain which of the two provides their users with better online and local privacy.

\section{Conflicts of Interest}

The authors declare no conflict of interest.

\section{Author Contributions}

Both authors, A. Mahlous and H. Mahlous contributed to the conceptualization, methodology, validation, data curation and writing-review.

Formal analysis, investigation, resources, writing - original draft preparation, supervision, and project administration were handled by A. Mahlous.

H. Mahlous took care of editing and visualization.

\section{References}

[1] https://us.norton.com/internetsecurity-privacywhat-is-private browsing.html

[2] https://www.ons.gov.uk/peoplepopulationandco mmunity/crimeandjustice/bulletins/crimeinengl andandwales/yearendingmarch2018

[3] K. Satvat, M. Forshaw, F. Hao, ansd E. Toreini, "On the privacy of private browsing - a forensic approach", Data Privacy Management and Autonomous Spontaneous Security, Springer, Berlin, Heidelberg, 2014.

[4] R. Montasari and P. Peltola, "Computer Forensic Analysis of Private Browsing Modes", In: Jahankhani H., Carlile A., Akhgar B., Taal A., Hessami A., Hosseinian-Far A. (eds) Global Security, Safety and Sustainability: Tomorrow's Challenges of Cyber Security. ICGS3 2015. Communications in Computer and Information Science, Vol. 534. Springer, Cham. https://doi.org/10.1007/978-3-319-23276-8_9, 2015.

[5] R. Md. Saidi, F. F. Saleh Udin, A. F. Zolkeplay, M. A. Arshad, F. Sappar "Analysis of Private Browsing Activities", In: Proc. of Yacob N., Mohd Noor N., Mohd Yunus N., Lob Yussof R., Zakaria S. (eds) Regional Conf. on Science, Technology and Social Sciences (RCSTSS 2016). Springer, Singapore, 2019.

[6] J. Oh, S. Lee, and S. Lee, "Advanced evidence collection and analysis of web browser activity", Digital Investigation, S62 - S70, 2011.

[7] D. N. Patil, B. B. Meshram "Web Browser Analysis for Detecting User Activities", In: $S a$ P., Bakshi S., Hatzilygeroudis I., Sahoo M. (eds) Recent Findings in Intelligent Computing Techniques. Advances in Intelligent Systems and Computing, Vol. 707. Springer, Singapore. 2019.

[8] D. J. Ohana and N. Shashidhar, "Do Private and Portable Web Browsers Leave Incriminating Evidence? A Forensic Analysis of Residual Artifacts from Private and Portable Web Browsing Sessions", In: Proc. of IEEE Security and Privacy Workshops, San Francisco, CA, pp. 135-142, 2013, doi: 10.1109/SPW.2013.18, 2013 
[9] N. A. Hassan "Web Browser and E-mail Forensics", In: Digital Forensics Basics. Apress, Berkeley, CA, 2019.

[10] G. Horsman, B. Findlay, J. Edwick, A. Asquith, K. Swannell, D. Fisher, A. Grieves, J. Guthrie, D. Stobbs, and P. McKain, "A forensic examination of web browser privacy-modes", In: Forensic Science International: Report, 2019.

[11] A. Nalawade, S. Bharne, and V. Mane, "Forensic analysis and evidence collection for web browser activity", In: Proc. of International Conf. on Automatic Control and Dynamic Optimization Techniques (ICACDOT), Pune, pp. 518-522, 2016, doi: 10.1109/ICACDOT.2016.7877639.

[12] H. Chivers, "Private browsing: a window of forensic opportunity", Digit. Investig. Vol. 11, No. 1, 2014.

[13] R. M. Gabet, K. C. Seigfried-Spellar, M. K. Rogers, "A comparative forensic analysis of privacy enhanced web browsers and private browsing modes of common web browsers", Int. J. Electron. Secur. Digit. Forensics, Vol. 10, No. 4, 356-371, 2018.

[14] C. Warren, E. El-Sheikh, NA. Le-Khac, "Privacy Preserving Internet Browsers: Forensic Analysis of Browzar", In: Daimi K. (eds) Computer and Network Security Essentials, Springer, Cham, 2018.

[15] A. Reed, M. Scanlon, N. A. Le-Khac, "Private Web Browser Forensics: A Case Study of the Epic Privacy Browser", 2018.

[16] https://www.forbes.com/sites/billybambrough/2 020/04/09/billions-of-google-chrome-usersnow-have-another-surprising-option

[17] https://netmarketshare.com/operating-systemmarket-share

[18] https://psusa.onthehub.com/WebStore/Welcome.aspx

[19] https://static1.squarespace.com/static/53cff120e 4b095a8ca26053d/t/53ff7b93e4b0e1f6ca3c99b d/1409252243947/nist-sp-800-88-rev1.pdf

[20] https://accessdata.com/productsservices/forensic-toolkit-ftk/ftkimager

[21] [https://www.autopsy.com

[22] [https://sourceforge.net/projects/regshot/

[23] https://www.magnetforensics.com/news/magne $\mathrm{t}$-forensics-releases-internet-evidence-finderv6-4/

[24] https://www.x-ways.net/winhex/

[25] https://www.similarweb.com/topwebsites/united-kingdom/

[26] https://www.tomshardware.com/news/how-tomanage-virtual-memory-pagefile-windows10,36929.html
[27] https://drfone.wondershare.com/dark-web/darkweb-browser.html 